\title{
A CANONICAL POWER SERIES EXPANSION FOR A SURFACE*
}

BX

ERNEST P. LANE

1. Introduction. In studying the projective differential geometry of an analytic surface in ordinary space it is frequently convenient to use a power series expansion for one non-homogeneous projective coordinate of a variable point on the surface in terms of the other two coordinates. Such an expansion can be reduced to various canonical forms by means of suitable choices of the projective coordinate system, that is, of the tetrahedron of reference and the unit point. The purpose of this paper is to deduce and apply such a canonical expansion, which does not seem to have been considered hitherto.

The form of the canonical expansion with which we are concerned was suggested in the first place by the exigencies adherent to the investigation of problems of a certain type which will be discussed more fully in $\S 3$. For the moment it is sufficient to say that the desideratum was a canonical expansion for which one edge of the tetrahedron of reference was an arbitrary nonasymptotic tangent at an ordinary non-parabolic point of a surface. This consideration led to the deduction of the canonical expansion which will be explained in detail in $\$ 2$. There the method followed is predominantly geometrical and the coordinate system employed is completely interpreted geometrically.

In $\S 4$ the connection between the canonical expansion introduced in this paper and a canonical expansion long known in the theory of conjugate nets is established, and some of the results of previous sections are applied in the theory of conjugate nets.

2. The canonical expansion. In this section a canonical power series expansion for a surface will be deduced. The geometrical significance of the successive steps in the work will be fully explained.

In projective space of three dimensions let us establish a point coordinate system, in which a point that has non-homogeneous coordinates $x, y, z$ also has homogeneous coordinates $x_{1}, x_{2}, x_{3}, x_{4}$ whose ratios are defined by placing

$$
x=\frac{x_{2}}{x_{1}}, \quad y=\frac{x_{3}}{x_{1}}, \quad z=\frac{x_{4}}{x_{1}} .
$$

\footnotetext{
* Presented to the Society, April 20, 1935; received by the editors December 5, 1934.
} 
Then let us consider an analytic surface $S$ whose equation is

$$
z=f(x, y) \text {. }
$$

It is well known that if the origin $O$, namely, the vertex of the tetrahedron of reference having non-homogeneous coordinates $0,0,0$, is an ordinary point on the surface $S$, and if the face $z=0$ of the tetrahedron is the tangent plane of $S$ at $O$, then the equation (1) of the surface can be expanded by Taylor's series into

$$
z=\alpha(x, y)+\beta(x, y)+\gamma(x, y)+\cdots,
$$

where $\alpha, \beta, \gamma, \cdots$ are forms which can be written as follows:

$$
\begin{aligned}
& \alpha(x, y)=a_{0} x^{2}+2 a_{1} x y+a_{2} y^{2}, \\
& \beta(x, y)=b_{0} x^{3}+3 b_{1} x^{2} y+3 b_{2} x y^{2}+b_{3} y^{3}, \\
& \gamma(x, y)=c_{0} x^{4}+4 c_{1} x^{3} y+6 c_{2} x^{2} y^{2}+4 c_{3} x y^{3}+c_{4} y^{4},
\end{aligned}
$$

the coefficients $a_{0}, \cdots, c_{4}, \cdots$ being constants. Let us assume that the origin $O$ is not a parabolic point. Then the discriminant $\Delta$ of the form $\alpha$, defined by

$$
\Delta=4\left(a_{0} a_{2}-a_{1}^{2}\right),
$$

is not zero, and the two asymptotic tangents at the origin, represented by the equation $\alpha=0$, are distinct.

The principal purpose of this section is to prove the following theorem:

By means of a suitable choice of the coordinate system, the equation (2) of a surface can be reduced to the canonical form

$$
z=x^{2}+y^{2}+x^{3}+b_{3} y^{3}+c_{0} x^{4}+c_{4} y^{4}+\cdots,
$$

the unwritten terms being of degree at least five, and the edge $y=z=0$ of the tetrahedron of reference being an arbitrary non-asymptotic tangent at the nonparabolic point $(0,0,0)$.

The proof begins with the observation that the coefficient $a_{1}$ can be made to vanish by taking for the edge $x=z=0$ of the tetrahedron of reference the tangent which is the harmonic conjugate of the edge $y=z=0$ with respect to the asymptotic tangents of the surface at the origin $O$. We shall suppose from now on that this has been done, so that $a_{1}=0$.

The next step in the proof consists in establishing the following lemma:

The coefficient $b_{1}$ vanishes if, and only if, the vertex $(0,0,1,0)$ corresponds to the face $y=0$ in Segre's correspondence, and similarly $b_{2}$ vanishes in case the vertex $(0,1,0,0)$ corresponds to the face $x=0$. 
Let us recall in this connection that Segre's correspondence associated with a point $O$ of a surface $S$ is by definition the correspondence between the osculating plane at $O$ of any curve $C$ on $S$ through $O$ and the ray-point of the curve $C$ at $O$, namely, the point of intersection of three tangent planes of $S$ constructed at $O$ and at two consecutive points on $C$. Segre studied* this correspondence, using a notation not differing essentially from ours, and proved that the equations of the correspondence can be written in the form

$$
X=\frac{\alpha(u, v) \alpha_{v}(u, v)}{\Delta \alpha(u, v)-J(u, v)}, \quad Y=-\frac{\alpha(u, v) \alpha_{u}(u, v)}{\Delta \alpha(u, v)-J(u, v)},
$$

the meanings of the new symbols appearing here having the following explanation. The symbols $X, Y$ represent the first two non-homogeneous coordinates of a point in the tangent plane of the surface $S$ at the origin $O$, the third coordinate being zero. Then $u$, $v$ are coordinates of a plane through $O$, the equation of this plane being

$$
z=v x-u y .
$$

Subscripts indicate partial differentiation, and $J$ is the jacobian of the forms $\alpha, \beta$ :

$$
J=\alpha_{u} \beta_{v}-\alpha_{v} \beta_{u} .
$$

Writing out equations (6) by means of equations (3) with $a_{1}=0$, placing $v=0$, and then allowing $u$ to become infinite, we find that the homogeneous coordinates of the point corresponding to the plane $y=0$ are $3 b_{1}, 0, a_{0}, 0$. Similarly, placing $u=0$ and then letting $v$ become infinite, we find that the point $\left(3 b_{2}, a_{2}, 0,0\right)$ corresponds to the plane $x=0$. One sees immediately that the first point is the vertex $(0,0,1,0)$ of the tetrahedron of reference if, and only if, $b_{1}=0$; similarly, the second point is the vertex $(0,1,0,0)$ in case $b_{2}=0$. Thus the lemma is established. We shall suppose from now on that $b_{1}=b_{2}=0$.

It is geometrically obvious that the face $x=0$ may still be any plane whatever through the tangent $x=z=0$, except the tangent plane, and likewise the face $y=0$ is an arbitrary plane through the tangent $y=z=0$. This fact can also be verified analytically in the following way. Let us make the transformation

$$
\begin{gathered}
x=\frac{x^{\prime}+B z^{\prime}}{1+2 a_{0} B x^{\prime}+2 a_{2} D y^{\prime}}, \quad y=\frac{y^{\prime}+D z^{\prime}}{1+2 a_{0} B x^{\prime}+2 a_{2} D y^{\prime}}, \\
z=\frac{z^{\prime}}{1+2 a_{0} B x^{\prime}+2 a_{2} D y^{\prime}},
\end{gathered}
$$

* Segre, Complementi alla teoria delle tangenti coniugate di una superficie, Rendiconti della Reale Accademia dei Lincei, (5), vol. 17 (1908), p. 405. 
from the old coordinates $x, y, z$ to new coordinates $x^{\prime}, y^{\prime}, z^{\prime}$, the coefficients $B, D$ being arbitrary. This transformation makes the two planes whose equations in the old coordinate system are

$$
x-B z=0, \quad y-D z=0
$$

become, respectively, the two faces $x^{\prime}=0$ and $y^{\prime}=0$ of the tetrahedron of reference of the new coordinate system. Moreover, this transformation carries the equation (2) of the surface, with $a_{1}=b_{1}=b_{2}=0$, into another equation of the same form in which the coefficients of the terms of the second and third degrees are absolutely unchanged. The calculations in support of this statement will not be reproduced here, but are straightforward, and complete the analytic verification aforementioned.

The transformation (9) can be used to reduce both of $c_{1}, c_{3}$ to zero. If the transformed coefficients of the terms of the fourth degree are calculated, it is found that, in particular, the coefficients $c_{1}, c_{3}$ obey the laws

$$
c_{1}^{\prime}=c_{1}-\frac{1}{2} a_{2} b_{0} D, \quad c_{3}^{\prime}=c_{3}-\frac{1}{2} a_{0} b_{3} B .
$$

Therefore, if $B, D$ have the values

$$
B=\frac{2 c_{3}}{a_{0} b_{3}}, \quad D=\frac{2 c_{1}}{a_{2} b_{0}},
$$

then the transformed coefficients $c_{1}^{\prime}, c_{3}^{\prime}$ are zero. The equations in the old coordinate system of the planes which must be taken as the faces $x^{\prime}=0$ and $y^{\prime}=0$ of the new tetrahedron in order to effect this reduction are found, by substituting the values of $B, D$ from (12) into (10), to be

$$
a_{0} b_{3} x-2 c_{3} z=0, \quad a_{2} b_{0} y-2 c_{1} z=0 .
$$

A geometric characterization of these planes is contained in the following lemma:

The first of the planes (13) is the plane that passes through the tangent line $x=z=0$ and touches the cone of Segre associated with the point $O$ of the surface; likewise the second of the planes (13) is the plane through the tangent line $y=z=0$ and touching the cone of Segre.

Beginning the proof, let us recall that the cone of Segre at a point $O$ of a surface $S$ is by definition the envelope of the planes through $O$ which are able to serve as stationary osculating planes at $O$ of curves on $S$ through $O$ and having at $O$ stationary ray-points. The equation of the cone of Segre, when the equation of the surface is in the general form (2), was shown* by Segre to be

\footnotetext{
* Segre, ibid.
} 


$$
\frac{1}{2} \alpha\left(\beta_{u u} \alpha_{v}-2 \beta_{u v} \alpha_{u} \alpha_{v}+\beta_{v v} \alpha_{u}^{2}-2 \Delta \beta\right)+2 \beta J-\alpha K=0,
$$

in which the arguments of the functions are the plane coordinates $u, v$, and the symbols $\Delta, J$ have the same meanings as in equations (6), while $K$ is the jacobian of the forms $\alpha, \gamma$ :

$$
K=\alpha_{u} \gamma_{v}-\alpha_{v} \gamma_{u} .
$$

To complete the proof it is sufficient to write out equation (14) by means of (3) with $a_{1}=b_{1}=b_{3}=0$, and then, on comparing each of equations (13) with equation (7), to show that the equation of the cone of Segre is satisfied by the values of $u, v$ given by

$$
u=0, \quad v=\frac{a_{0} b_{3}}{2 c_{3}},
$$

and is also satisfied by

$$
u=-\frac{a_{2} b_{0}}{2 c_{1}}, \quad v=0 .
$$

We shall suppose from now on that $c_{1}=c_{3}=0$.

The position of the vertex $(0,0,0,1)$ on the edge $x=y=0$ of the tetrahedron of reference is still at our disposal. It will now be shown that this vertex can be chosen so as to reduce $c_{2}$ to zero. In fact, the transformation of coordinates

$$
x=\frac{x^{\prime}}{1+H z^{\prime}}, \quad y=\frac{y^{\prime}}{1+H z^{\prime}}, \quad z=\frac{z^{\prime}}{1+H z^{\prime}},
$$

in which the coefficient $H$ is arbitrary, makes the point whose coordinates in the old coordinate system are $H, 0,0,1$ become the vertex $(0,0,0,1)$ of the tetrahedron of reference of the new coordinate system. Moreover, this transformation changes the equation of the surface into another equation of the same form in which the coefficients of the terms of the second and third degrees are absolutely unchanged, and in which the coefficients $c_{1}^{\prime}$ and $c_{3}^{\prime}$ are still zero, while the coefficient $c_{2}^{\prime}$ is given by the formula

$$
c_{2}^{\prime}=c_{2}-\frac{1}{3} a_{0} a_{2} H \text {. }
$$

Therefore, if $H$ has the value given by

$$
H=\frac{3 c_{2}}{a_{0} a_{2}},
$$

then $c_{2}^{\prime}=0$. The homogeneous coordinates in the old coordinate system of the 
point $V$ which must be taken as the vertex $(0,0,0,1)$ of the tetrahedron of reference of the new coordinate system in order to effect this reduction are

$$
\frac{3 c_{2}}{a_{0} a_{2}}, 0,0,1 \text {. }
$$

A simple geometric construction for the point $V$ is certainly desirable. The following discussion shows how to construct this point by means of its relations to certain other points on the line $x=y=0$, namely, the points distinct from the origin $O$, in which this line intersects certain quadrics associated with the point $O$ of the surface $S$.

First of all, the equation of the canonical quadric of Wilczynski can be found by means of the characteristic property discovered* by Bompiani to the effect that this quadric is an asymptotic osculating quadric of a curve on a surface which has an inflexion at the point under consideration. The details of the calculation of the equation need not be reproduced here, but the required equation of the canonical quadric of Wilczynski turns out to be

$$
z=a_{0} x^{2}+a_{2} y^{2}+\frac{3}{4} \frac{b_{0}}{a_{0}} x z+\frac{3}{4} \frac{b_{3}}{a_{2}} y z+W z^{2},
$$

the coefficient $W$ being given by the formula

$$
W=\frac{3}{8}\left[\frac{c_{0}}{a_{0}{ }^{2}}+\frac{2 c_{2}}{a_{0} a_{2}}+\frac{c_{4}}{a_{2}{ }^{2}}-\frac{3}{4}\left(\frac{b_{0}{ }^{2}}{a_{3}{ }^{3}}+\frac{b_{3}{ }^{2}}{a_{2}{ }^{3}}\right)\right] .
$$

The equation of the quadric of Lie can be found by means of its definition as the quadric determined by three consecutive asymptotic tangents of one family constructed at the point $O$ under consideration and at two consecutive points on the asymptotic curve of the other family through $O$. The required equation of the quadric of Lie has the same form as equation (20) except that the coefficient $W$ is replaced by a coefficient $L$ given by

$$
L=\frac{3}{8}\left[\frac{c_{0}}{a_{0}^{2}}+\frac{2 c_{2}}{a_{0} a_{2}}+\frac{c_{4}}{a_{2}^{2}}-\frac{15}{16}\left(\frac{b_{0}^{2}}{a_{0}^{2}}+\frac{b_{3}^{2}}{a_{2}{ }^{2}}\right)\right] .
$$

As a matter of fact, the equation (20) with $W$ arbitrary represents any quadric of Darboux.

The equation of an asymptotic osculating quadric at the point $O$ for the curve cut on the surface by the plane $y=0$ can be found from its definition as the quadric determined by three consecutive asymptotic tangents of one

* Bompiani, Fascio di quadriche di Darboux e normale proiettiva in un punto di una superficie, Rendiconti della Reale Accademia dei Lincei, (6), vol. 6 (1927), p. 187. 
family constructed at $O$ and two consecutive points on the curve. We find that the equations of the two asymptotic osculating quadrics at the point $O$ for the curve $y=0$ can be written together in the form

$$
z=a_{0} x^{2}+a_{2} y^{2}+\frac{9}{4} \frac{b_{0}}{a_{0}} x z \mp \frac{15}{4} \frac{b_{0}}{a_{0} w} y z+B z^{2},
$$

where $w$ is defined by

$$
w=\left(-\frac{a_{0}}{a_{2}}\right)^{1 / 2}
$$

and the coefficient $B$ is given by

$$
B=\frac{3}{2}\left(\frac{c_{0}}{a_{0}^{2}}-\frac{c_{2}}{a_{0} a_{2}}-\frac{9}{8} \frac{b_{0}^{2}}{a_{0}^{8}}\right) .
$$

The analogous equation of the two asymptotic osculating quadrics at the point $O$ for the curve $x=0$ can be written by symmetry and is

$$
z=a_{0} x^{2}+a_{2} y^{2}+\frac{9}{4} \frac{b_{3}}{a_{2}} y z \mp \frac{15}{4} \frac{b_{3} w}{a_{2}} x z+K z^{2},
$$

where the coefficient $K$ is given by

$$
K=\frac{3}{2}\left(\frac{c_{4}}{a_{2}^{2}}-\frac{c_{2}}{a_{0} a_{2}}-\frac{9}{8} \frac{b_{3}{ }^{2}}{a_{2}^{3}}\right) .
$$

The coordinates of the point distinct from $O$ in which the line $x=y=0$ pierces the canonical quadric of Wilczynski are $W, 0,0,1$. We may speak of this point as the point $W$, since $W$ is a non-homogeneous coordinate of this point on the line $x=y=0$. With similar terminology in analogous cases, the following construction locates the point $H$ which we are seeking to characterize.

Let $P$ be the harmonic conjugate of the point $W$ with respect to the points $L$ and $O$. Let $Q$ be the harmonic conjugate of $O$ with respect to $B$ and $K$. Then the point $H$ is the harmonic conjugate of $Q$ with respect to $P$ and $O$.

The proof consists in showing first that the coordinates $P, Q$ of the points $P, Q$ are given by the formulas

$$
\begin{aligned}
& P=\frac{3}{8}\left[\frac{c_{0}}{a_{0}{ }^{2}}+\frac{2 c_{2}}{a_{0} a_{2}}+\frac{c_{4}}{a_{2}{ }^{2}}-\frac{9}{8}\left(\frac{b_{0}{ }^{2}}{a_{0}{ }^{3}}+\frac{b_{3}{ }^{2}}{a_{2}{ }^{3}}\right)\right], \\
& Q=\frac{3}{4}\left[\frac{c_{0}}{a_{0}{ }^{2}}-\frac{2 c_{2}}{a_{0} a_{2}}+\frac{c_{4}}{a_{2}{ }^{2}}-\frac{9}{8}\left(\frac{b_{0}{ }^{2}}{a_{0}{ }^{3}}+\frac{b_{3}{ }^{2}}{a_{2}{ }^{3}}\right)\right],
\end{aligned}
$$


and in then showing that the point $H$, given by equation (18), is such that the cross ratio of the points $O, P, Q, H$ in the order written is -1 .

The vertices of the tetrahedron of reference are now completely specified when the point $O$ and the tangent $y=z=0$ are given. We shall suppose from now on that $c_{2}=0$, and have shown how to reduce the equation (2) of a surface to the form

$$
z=a_{0} x^{2}+a_{2} y^{2}+b_{0} x^{3}+b_{3} y^{3}+c_{0} x^{4}+c_{4} y^{4}+\cdots,
$$

the unwritten terms being of degree at least five.

There remains the transformation of unit point,

$$
x=l x^{\prime}, \quad y=m y^{\prime}, \quad z=n z^{\prime},
$$

the coefficients $l, m, n$ being arbitrary. If this transformation is effected on equation (29), if the transformed equation is solved for $z^{\prime}$ and the coefficients $a_{0}^{\prime}, a_{2}^{\prime}, b_{0}^{\prime}$ are equated to unity, the following conditions on $l, m, n$ are obtained:

$$
a_{0} l^{2}=a_{2} m^{2}=b_{0} l^{3}=n .
$$

Solving these equations for $l, m, n$, we find

$$
l=\frac{a_{0}}{b_{0}}, \quad m=\frac{a_{0}}{b_{0}}\left(\frac{a_{0}}{a_{2}}\right)^{1 / 2}, \quad n=\frac{a_{0}^{3}}{b_{0}^{2}},
$$

and thus arrive at the following lemma:

The unit point can be chosen so as to make $a_{0}=a_{2}=b_{0}=1$.

The theorem stated at the beginning of this section has now been proved. However, it still remains to describe geometrically the point whose coordinates in the old coordinate system are

$$
\frac{a_{0}}{b_{0}}, \quad \frac{a_{0}}{b_{0}}\left(\frac{a_{0}}{a_{2}}\right)^{1 / 2}, \quad \frac{a_{0}^{3}}{b_{0}^{2}},
$$

which becomes the unit point in the new coordinate system. This point will be located as the intersection of three planes.

First of all, this point obviously lies on one of the two planes whose equation is

$$
a_{0} x^{2}-a_{2} y^{2}=0 .
$$

These are the planes determined by the line $x=y=0$ and the associate conjugate tangents of the tangents $x=z=0$ and $y=z=0$, that is, the tangents which separate the latter harmonically and also separate the asymptotic tangents harmonically. 
To find a second plane containing the point (33) let us observe that the equation of the quadric of Moutard for the tangent $y=z=0$ is

$$
z=a_{0} x^{2}+a_{2} y^{2}+\frac{b_{0}}{a_{0}} x z+\left(\frac{c_{0}}{a_{0}^{2}}-\frac{b_{0}{ }^{2}}{a_{0}^{3}}\right) z^{2},
$$

as can be verified by finding the locus of the osculating conics at the point $O$ of the curves of section of the surface made by planes through this tangent. The equation of the tangent plane of this quadric at the point $(0,1,0,0)$ is

$$
2 a_{0}^{2} x+b_{0} z=0 .
$$

The harmonic conjugate of the plane $x=0$ with respect to the tangent plane, $z=0$, and the plane (36) has the equation

$$
a_{0}^{2} x+b_{0} z=0 .
$$

Finally, the harmonic conjugate of the plane (37) with respect to the planes $z=0$ and $x=0$ is represented by the equation

$$
a_{0}^{2} x-b_{0} z=0,
$$

and obviously contains the point (33).

A third plane containing this point can be found in the following way. The cubic surface whose equation is

$$
z=a_{0} x^{2}+a_{2} y^{2}+b_{0} x^{3}+b_{3} y^{3}
$$

is completely characterized by the following properties: it has third-order contact with the surface at the point $O$; it has a unode at the point $(0,0,0,1)$; and its uniplane is the plane $x_{1}=0$. Now the polar plane of the point $(0,1$, $0,0)$ with respect to the cubic (39) has the equation

$$
a_{0} x_{1}+3 b_{0} x_{2}=0
$$

in homogeneous coordinates. The equation of the harmonic conjugate of this plane with respect to the planes $x_{1}=0$ and $x_{2}=0$ is

$$
a_{0} x_{1}-3 b_{0} x_{2}=0 .
$$

The equation of the harmonic conjugate of the plane (41) with respect to the plane $x_{1}=0$ and the plane (40) is

$$
a_{0} x_{1}+b_{0} x_{2}=0 \text {. }
$$

Finally, the harmonic conjugate of the plane (42) with respect to the planes $x_{1}=0$ and $x_{2}=0$ has the equation

$$
a_{0} x_{1}-b_{0} x_{2}=0,
$$


and evidently contains the point (33), whose geometric description is now complete.

Two remarks may be adjoined. Since the derivative $\partial^{2} z / \partial x \partial y$ calculated from equation (39) vanishes identically, it follows that the pencils of planes whose axes are the lines $x=x_{1}=0$ and $y=x_{1}=0$ cut on the cubic surface (39) a conjugate net. In the second place, a similar remark can be made about the quadric surface whose equation is

$$
z=a_{0} x^{2}+a_{2} y^{2},
$$

and which is completely characterized by the following properties. It has second-order contact with the surface at the point $O$; the lines $z=x_{1}=0$ and $x=y=0$ are reciprocal polars with respect to it; and it passes through the point $(0,0,0,1)$.

3. Plane sections through a tangent. The canonical expansion deduced in the preceding section was designed to be used in solving problems of a certain type which may be identified as follows. Let us pass a plane through a non-asymptotic tangent at a point $O$ of a surface and consider geometric elements projectively associated with the point $O$ of the plane curve of intersection of the surface and plane. The problem is to discuss the loci of these elements when the plane turns around the tangent line.

Moutard was probably the first to solve a projective problem of this type when he proved that the locus of the osculating conics of the plane curves of section made by planes through a tangent is a quadric surface, which now bears his name. Kubota and Su have also solved* projective problems of this type, by showing among other things that the locus of the projective normals is a cubic cone of the third order. The purpose of this section is to continue and extend the investigations of Kubota and Su. Since the projective normal at a point of a plane curve is a nodal tangent of the eight-point nodal cubic, it is proposed here to consider all the eight-point cubics, and the osculating cubic, of a plane curve of section of a surface, and to investigate their loci and the loci of various points and lines associated with them, when the plane turns around the tangent.

Let us consider a surface $S$ whose equation in non-homogeneous projective coordinates has been reduced to the canonical form

$$
\begin{aligned}
z= & x^{2}+y^{2}+x^{3}+A y^{3}+B x^{4}+C y^{4}+D x^{5}+E x^{4} y+F x^{3} y^{2} \\
& +G x^{2} y^{3}+\cdots+H x^{6}+I x^{5} y+J x^{4} y^{2}+\cdots+K x^{7}+L x^{6} y \\
& +\cdots+M x^{8}+\cdots,
\end{aligned}
$$

* Kubota and Su, Eine Bemerkung zur Projektivdifferentialgeometrie der Flächen, Science Reports of the Tôhoku Imperial University, (1), vol. 19 (1930), p. 293. 
all the coefficients of which are absolute invariants of the surface, the terms written being the only ones that will be needed hereinafter. The equation

$$
y=n z
$$

represents a plane $\pi$ through the line $t$ whose equations are $y=z=0$ and which is an ordinary tangent of the surface $S$ at the origin $O(0,0,0)$. The plane $\pi$ cuts through the surface $S$ in a curve whose projection from the point $(0,0,0,1)$ onto the tangent plane, $z=0$, of $S$ at $O$ is a curve $C$. The equation, in the tangent plane, of the curve $C$ is found, by eliminating $z$ between equations (45), (46), to be

$$
\begin{aligned}
\frac{y}{n}= & x^{2}+y^{2}+x^{3}+A y^{3}+B x^{4}+C y^{4}+D x^{5}+E x^{4} y+F x^{3} y^{2} \\
& +G x^{2} y^{3}+\cdots+H x^{6}+I x^{5} y+J x^{4} y^{2}+\cdots+K x^{7}+L x^{6} y \\
& +\cdots+M x^{8}+\cdots .
\end{aligned}
$$

If this equation is solved for $y$ as a power series in $x$, the result to terms of the eighth degree is

$$
y=n\left(x^{2}+x^{3}+b_{4} x^{4}+b_{5} x^{5}+b_{6} x^{6}+b_{7} x^{7}+b_{8} x^{8}+\cdots\right),
$$

the coefficients $b_{4}, \cdots, b_{8}$ being defined by the formulas

$$
\begin{aligned}
b_{4}= & n^{2}+B, \\
b_{5}= & 2 n^{2}+D, \\
b_{6}= & 2 n^{4}+A n^{3}+(2 B+1) n^{2}+E n+H, \\
b_{7}= & 6 n^{4}+3 A n^{3}+(2 B+2 D+F) n^{2}+(E+I) n+K, \\
b_{8}= & 5 n^{6}+5 A n^{5}+(6 B+C+6) n^{4}+(3 A+3 E+G+3 A B) n^{3} \\
& +\left(2 D+2 F+2 H+J+B^{2}\right) n^{2}+(I+L+B E) n+M .
\end{aligned}
$$

The equation of the osculating conic of the curve $C$ at the origin $O$ is found by writing the most general equation of the second degree in $x, y$ and then demanding that this equation be satisfied by the series (48) for $y$ identically in $x$ as far as the term in $x^{4}$. The result is

$$
y-n x^{2}-x y-R \frac{y^{2}}{n}=0,
$$

where $R$ is a polynomial defined by placing

$$
R=n^{2}+B-1 \text {. }
$$

The equation of the quadric of Moutard for the tangent $t$ at the point $O$ is found, by eliminating $n$ between equations (46), (50), to be 


$$
z-x^{2}-y^{2}-x z-(B-1) z^{2}=0 .
$$

Let the left member of equation (50) be denoted by $Q$, so that

$$
Q=y-n x^{2}-x y-R \frac{y^{2}}{n} .
$$

Then substitution of the series (48) for $y$ yields, to terms of the fifth degree,

$$
Q=\operatorname{Sn} x^{5}+\cdots,
$$

the coefficient $S$ being defined by the formula

$$
S=-n^{2}-3 B+D+2 .
$$

The two values of $n$ which make $S$ vanish yield, when substituted in equation (46), the equations of the two planes producing sections of the surface which are hyperosculated by their osculating conics. These equations can be written together in the form.

$$
y^{2}-(-3 B+D+2) z^{2}=0,
$$

which establishes the following theorem:

At a point $O$ of a surface $S$ the two planes through a tangent $t$ which produce sections which are hyperosculated by their osculating conics separate harmonically the tangent plane and the plane through the tangent $t$ which touches the cone of Segre.

The equation of all eight-point cubics at the point $O$ of the curve $C$ is found by writing the most general equation of the third degree in $x, y$ and then demanding that this equation be satisfied by the series (48) for $y$ identically in $x$ as far as the term in $x^{7}$. The result can be written in the form

$$
S\left[y-n x^{2}-n x^{3}-(R+1) x^{2} y-(S+2 R) x \frac{y^{2}}{n}-\left(V+2 S+R^{2}\right) \frac{y^{3}}{n^{2}}\right]
$$

$$
-W Q \frac{y}{n}+h\left[\left(S x-V \frac{y}{n}\right) Q-S^{2} \frac{y^{3}}{n^{2}}\right]=0,
$$

in which $h$ is a parameter and $V, W$ are two polynomials defined by the formulas

$$
\begin{aligned}
V= & A n^{3}-(2 B-3) n^{2}+E n+10 B-4 D+H-2 B^{2}-5 \\
W= & -n^{4}-(D-F-2) n^{2}-(2 E-I) n-5 B+5 D-3 H+K \\
& -3 B D+5 B^{2} .
\end{aligned}
$$

The equation of the eight-point nodal cubic is 


$$
\left(S x-V \frac{y}{n}\right) Q-S^{2} \frac{y^{3}}{n^{2}}=0 .
$$

The nodal tangents of this cubic are the tangent $t$, whose equation is $y=0$, and the projective normal, whose equation is

$$
S x-V \frac{y}{n}=0 .
$$

The equation of the flex-ray, that is, the line containing the three inflexions of the eight-point nodal cubic, is found to be

$$
S^{2}-\left(2 V S+S^{2}\right) x+\left(V^{2}-R S^{2}\right) \frac{y}{n}=0 .
$$

The flex-ray is known to be tangent to the osculating conic (50) at the point of intersection of the flex-ray (61) and the projective normal (60), namely, the point

$$
\left(\frac{V S}{V^{2}+V S+R S^{2}}, \frac{S^{2} n}{V^{2}+V S+R S^{2}}\right) \text {. }
$$

Moreover, the flex-ray intersects the tangent $t$ at the point

$$
\left(\frac{S}{2 V+S}, 0\right)
$$

All the eight-point cubics (57) intersect, besides at the origin $O$, also in the Halphen point,

$$
\begin{aligned}
& \left(\frac{S T\left(V T-S^{4}\right)}{\left(V T-S^{4}\right)^{2}+S T\left(V T-S^{4}\right)+T^{2}\left(R S^{2}-T\right)},\right. \\
& \left.\frac{S^{2} T^{2} n}{\left(V T-S^{4}\right)^{2}+S T\left(V T-S^{4}\right)+T^{2}\left(R S^{2}-T\right)}\right)
\end{aligned}
$$

wherein $T$ is a polynomial defined by the formula

$$
T=V^{2}+2 V S+2 R S^{2}-S W .
$$

The equation of the line joining the origin to the Halphen point is

$$
S T x-\left(V T-S^{4}\right) \frac{y}{n}=0 .
$$

The eight-point cubic of Sannia is by definition that eight-point cubic which passes through the point (63), or equally well, that eight-point cubic 
which passes through the point (62). Either way one finds that for the eightpoint cubic of Sannia the parameter $h$ in equation (57) has the value

$$
h=-2\left(\frac{V}{S}+1\right) \text {. }
$$

With this value of $h$ in equation (57), the equation of the eight-point cubic of Sannia can be written in the form

$$
\begin{gathered}
{\left[S^{2}-\left(2 V S+S^{2}\right) x+\left(V^{2}-R S^{2}\right) \frac{y}{n}+T \frac{y}{n}\right] Q} \\
-S^{2}\left(S x-V \frac{y}{n}\right) \frac{y^{2}}{n}=0 .
\end{gathered}
$$

Moreover, it is evident on inspection that equation (53) defining $Q$ is equivalent to

$$
S^{2} Q=\left[S^{2}-\left(2 V S+S^{2}\right) x+\left(V^{2}-R S^{2}\right) \frac{y}{n}\right] y-n\left(S x-V \frac{y}{n}\right)^{2} .
$$

Therefore the flex-ray (61) meets the eight-point cubic of Sannia, besides in the points (62), (63), also in the point where the flex-ray intersects the line (66) joining the origin to the Halphen point, namely in the point

$$
\begin{aligned}
& \left(\frac{S\left(V T-S^{4}\right)}{\left(V T-S^{4}\right)(2 V+S)-T\left(V^{2}-R S^{2}\right)}\right. \\
& \left.\qquad \frac{S^{2} T n}{\left(V T-S^{4}\right)(2 V+S)-T\left(V^{2}-R S^{2}\right)}\right) .
\end{aligned}
$$

The osculating cubic is represented by equation (57) when the parameter $h$ has the value obtained by demanding that $(57)$ be satisfied by the series (48) for $y$ as far as the term in $x^{8}$. Thus the value of $h$ for the osculating cubic is found to be given by

$$
\begin{aligned}
T h= & S\left[A n^{5}-(B-C+1) n^{4}-(E-G+A B) n^{3}\right. \\
& +\left(2 F+J-2 H+2 B^{2}+3\right) n^{2}-(3 E-I-L+3 B E) n \\
& \left.-6 B+6 D-3 H+M+6 B D-4 B H-B^{2}-2 D^{2}+3 B^{3}\right] \\
& -(V+4 S) W .
\end{aligned}
$$

The right member of this equation reduces to a polynomial of the sixth degree in $n$, as does $T$ also.

The osculating conic (50) intersects any eight-point cubic (57), besides at the origin also at the point 


$$
\begin{aligned}
\left(\frac{-S(V+2 S+h S)}{(V+2 S+h S)^{2}-S(V+2 S+h S)+R S^{2}}\right. \\
\left.\frac{S^{2} n}{(V+2 S+h S)^{2}-S(V+2 S+h S)+R S^{2}}\right) .
\end{aligned}
$$

For the value of $h$ given by (67) this point becomes the point (62), and for the value of $h$ given by (70) this point is the residual intersection of the osculating conic and the osculating cubic. The equation of the line joining the origin to the point (71) is obviously

$$
S x+(V+2 S+h S) \frac{y}{n}=0 .
$$

It is now easy to write the equation of the cone of Kubota and $S u$, which is the locus of the projective normals. Elimination of $n$ between equations (46), (60) yields the desired equation,

$$
\begin{gathered}
{\left[y^{2}-(-3 B+D+2) z^{2}\right] x+A y^{3}+(-2 B+3) y^{2} z+E y z^{2}} \\
+\left(10 B-4 D+H-2 B^{2}-5\right) z^{3}=0 .
\end{gathered}
$$

The form of this equation makes it evident at once that the tangent $t$, $y=z=0$, is a double line of this cone, as remarked* by Kubota and Su. Moreover, the equation of the nodal tangent planes of the cone along this line is obtained by setting equal to zero the coefficient of $x$ in equation (73), and is precisely equation (56). Thus the following theorem is proved.

The nodal tangent planes of the cone of Kubota and Su along its double line are the planes through this line that cut the surface in curves which are hyperosculated by their osculating conics.

Furthermore, the cone of Kubota and Su cuts the tangent plane, $z=0$, in the line

$$
x+A y=0,
$$

besides the tangent $t$. Finally, the plane containing the three inflexional generators of the cone is represented by the equation

$$
\begin{aligned}
& 4(3 B-D-2) x+[E+3 A(3 B-D-2)] y \\
& \quad+\left[(2 B-3)(2-3 B+D)+3\left(10 B-4 D+H-2 B^{2}-5\right)\right] z=0 .
\end{aligned}
$$

For the purpose of facilitating calculation it is convenient to introduce seven constants $a, b, c, d, e, f, g$ defined by the following formulas:

* Kubota and Su, ibid., p. 300. 


$$
\begin{aligned}
& a=B-1, \\
& b=-3 B+D+2, \\
& c=-2 B+3 \\
& d=10 B-4 D+H-2 B^{2}-5, \\
& e=-D+F+2, \\
& f=-2 E+I \\
& g=-5 B+5 D-3 H+K-3 B D+5 B^{2} .
\end{aligned}
$$

Then one can rewrite quite simply the definitions of $R, S, V, W$ :

$$
\begin{aligned}
R & =n^{2}+a, \\
S & =-n^{2}+b, \\
V & =A n^{3}+c n^{2}+E n+d, \\
W & =-n^{4}+e n^{2}+f n+g .
\end{aligned}
$$

Actual calculation now yields

$$
T=\left(1+A^{2}\right) n^{6}-4 A(B-1) n^{5}+\alpha n^{4}+\beta n^{3}+\gamma n^{2}+\delta n+\epsilon,
$$

where $\alpha, \beta, \gamma, \delta, \epsilon$ are defined by

$$
\begin{aligned}
\alpha & =2 a-3 b-2 c+e+c^{2}+2 A E, \\
\beta & =2(A b+A d+E c-E)+f, \\
\gamma & =2 b c+2 c d-2 d+2 b^{2}-4 a b-b e+g+E^{2}, \\
\delta & =2 E(b+d)-b f, \\
\epsilon & =2 b d+2 a b^{2}-b g+d^{2} .
\end{aligned}
$$

The values of $n$ which satisfy the equation $T=0$ make the Halphen point (64) coincide with the origin, which is then a coincidence point on the corresponding curve of section. Thus one arrives at the following theorem:

Through an ordinary tangent at a point $O$ of a surface there pass six planes which cut the surface in curves having a coincidence point at $O$.

It is known that at a coincidence point the eight-point nodal cubic is the osculating cubic. It is suggested to inquire whether the eight-point cubic of Sannia can be the osculating cubic. Demanding that equation (68) be satisfied by the power series (48) for $y$ as far as the term in $x^{8}$, we find that $n$ must be a solution of an equation of the ninth degree,

$$
A\left(1+A^{2}\right) n^{9}+\cdots+9=0,
$$

the unwritten terms being not needed for our purposes. Thus the following theorem is established. 
Through an ordinary tangent at a point $O$ of a surface there pass nine planes which cut the surface in curves each of which is osculated at $O$ by its eight-point cubic of Sannia.

Besides the projective normal there are other lines whose loci are of interest. For example, the locus of the flex-ray is an algebraic ruled surface of the sixth order, as remarked* by Kubota and Su, whose equation is found, by eliminating $n$ from (61), (46), to be

$$
\begin{aligned}
& z\left(y^{2}-b z^{2}\right)^{2}-x\left[-2 A y^{5}+(4 B-5) y^{4} z+2(A b-E) y^{3} z^{2}\right. \\
& \left.\quad+2(b c-b-d) y^{2} z^{3}+2 E b y z^{4}+\left(2 b d+b^{2}\right) z^{5}\right]+\left(A^{2}-1\right) y^{6} \\
& \quad+2 A(3-2 B) y^{5} z+\left(2 A E+c^{2}+2 b-a\right) y^{4} z^{2}+2(A d+E c) y^{3} z^{3} \\
& \quad+\left(E^{2}+2 c d-b^{2}+2 a b\right) y^{2} z^{4}+2 E d y z^{5}+\left(d^{2}-a b^{2}\right) z^{6}=0 .
\end{aligned}
$$

This surface has the tangent $t, y=z=0$, for quintuple line, and intersects the tangent plane, $z=0$, also in the line whose equation is

$$
2 A x+\left(A^{2}-1\right) y=0 .
$$

The locus of the line from the origin to the Halphen point is found from (66), (46) to be an algebraic cone of the ninth order, and the locus of the line from the origin to the point of intersection of the osculating conic and osculating cubic is found from (72), (46), (70) to be also an algebraic cone of the ninth order. The equations of these cones can be written without difficulty, but will not be included here.

The loci of three cubic curves which we have considered will now be discussed. The equation of the locus of the eight-point nodal cubic is found, by eliminating $n$ from equations (59), (46), to be

$$
\begin{gathered}
\left(z-x^{2}-y^{2}-x z-a z^{2}\right)\left[x\left(y^{2}-b z^{2}\right)+A y^{3}+c y^{2} z+E y z^{2}+d z^{3}\right] \\
+z\left(y^{2}-b z^{2}\right)^{2}=0 .
\end{gathered}
$$

Therefore this locus is an algebraic surface of the fifth order, which intersects the tangent plane, $z=0$, in the asymptotic tangents, $x^{2}+y^{2}=0$, and in the line (74), besides having the tangent $t, y=z=0$, for double line. Moreover, this surface intersects the quadric of Moutard (52) in the asymptotic tangents, and is tangent to the quadric of Moutard along the conics cut on this quadric by the planes (56). The locus of the eight-point cubic of Sannia is found from (68), (46) to be an algebraic surface of the eighth order, and the locus of the osculating cubic is found from (57), (46), (70) to be an algebraic surface of the twelfth order, but the equations of these surfaces will not be included here.

* Kubota and Su, ibid., p. 300. 
There are four points whose loci will now be briefly examined, namely, the intersection of the flex-ray and the projective normal, the Halphen point, the intersection of the flex-ray and the line from the origin to the Halphen point, and finally the intersection of the osculating conic and the osculating cubic. The parametric equations of the locus of the first point are obtained by setting $x$ and $y$ respectively equal to the coordinates given in (62), by adjoining the equation $z=y / n$, and by then replacing the polynomials $V, S, R$ by their expressions in terms of $n$ given in (77). Thus one finds that the locus of the intersection of the flex-ray and the projective normal is a unicursal curve of order six, as Kubota and $\mathrm{Su}^{*}$ have remarked. Similarly, one finds that the loci of the other three points in the order just named are unicursal curves of orders seventeen, twelve, and eighteen. A more detailed study of the properties and relations of the loci mentioned in this section is reserved for another occasion.

4. Applications to conjugate nets. The theory developed in the preceding section has interesting connections with the theory of conjugate nets. The coordinates $x$ of a point on a surface referred to a conjugate net in ordinary space, and the coordinates $y$ of the point which is the harmonic conjugate of the point $x$ with respect to the foci of the axis of the point $x$, satisfy a system of equations $\dagger$ of the form

$$
\begin{aligned}
& x_{u u}=p x+\alpha x_{u}+L y, \\
& x_{u v}=c x+a x_{u}+b x_{v}, \\
& x_{v v}=q x+\delta x_{v}+N y .
\end{aligned}
$$

The ray-points of the net at the point $x$ are given by the formulas

$$
x_{1}=x_{v}-a x, \quad x_{-1}=x_{u}-b x .
$$

Some of the invariants of the net are given by

$$
\begin{aligned}
8 \mathfrak{B}^{\prime} & =4 a-2 \delta+(\log r)_{v}, & & r=N / L, \\
8 \mathcal{S}^{\prime} & =4 b-2 \alpha-(\log r)_{u}, & & \\
H & =c+a b-a_{u}, & & \mathfrak{Q}=c+a b+b_{v}-\delta_{u}, \\
K & =c+a b-b_{v}, & & \Re=c+a b+a_{u}-\alpha_{v} .
\end{aligned}
$$

If the four points $x, x_{-1}, x_{1}, y$ are used as the vertices of the tetrahedron of reference of a local coordinate system, it is not difficult to calculate by familiar methods a power series expansion for one non-homogeneous co-

* Kubota and Su, ibid., p. 300.

$\dagger$ Lane, Projective Differential Geometry of Curves and Surfaces, University of Chicago Press, 1932, p. 138. 
ordinate $z$ of a point on the surface in terms of the other two coordinates $x, y$. Thus one obtains, to terms of the fourth order,

$$
\begin{gathered}
z=\frac{1}{2}\left(L x^{2}+N y^{2}\right)+\frac{4}{3}\left(L \mathscr{S}^{\prime} x^{3}+N \mathscr{B}^{\prime} y^{3}\right)+c_{0} x^{4}+4 c_{1} x^{8} y \\
+4 c_{3} x y^{3}+c_{4} y^{4}+\cdots,
\end{gathered}
$$

where the coefficients $c_{0}, c_{1}, c_{3}, c_{4}$ are defined by

$$
\begin{array}{ll}
c_{0}=\frac{1}{3} L \mathfrak{S}^{\prime}\left[12 \mathfrak{S}^{\prime}+\left(\log \mathfrak{S}^{\prime} r^{1 / 2}\right)_{u}\right], & 4 c_{1}=\frac{1}{6} L(H-\mathfrak{S}), \\
c_{4}=\frac{1}{3} N \mathfrak{B}^{\prime}\left[12 \mathfrak{B}^{\prime}+\left(\log \mathfrak{B}^{\prime} r^{-1 / 2}\right)_{v}\right], & 4 c_{3}=\frac{1}{6} N(K-\Re),
\end{array}
$$

and the coefficient of $x^{2} y^{2}$ is zero. G. M. Green calculated* an expansion essentially the same as this one to terms of the third order, and showed that the coefficient of $x^{2} y^{2}$ is zero, but apparently did not concern himself with the other terms of the fourth order.

Comparison of the expansion (84) with the expansion (29) leads to interesting results. Equations (13) show that the equations of the planes which must be taken as two faces of a new tetrahedron of reference to reduce (84) to precisely the form of (29) are

$$
8 L \mathfrak{B}^{\prime} x-(K-\Re) z=0, \quad 8 N \overleftarrow{\complement}^{\prime} y-(H-\mathfrak{E}) z=0 .
$$

The points which are used in place of the ray-points as vertices of the new tetrahedron are the points

$$
\frac{K-\Re}{8 \mathfrak{B}^{\prime}} x+x_{-1}, \quad \frac{H-\mathfrak{E}}{8 \mathfrak{E}^{\prime}} x+x_{1},
$$

and the line which is used in place of the axis as an edge of the new tetrahedron joins the point $x$ to the point

$$
\frac{K-\Re}{8 L \mathfrak{B}^{\prime}} x_{-1}+\frac{H-\mathfrak{S}}{8 L \mathscr{S}^{\prime}} x_{1}+y .
$$

It is known $\dagger$ that the ray curves and the axis curves of a non-harmonic conjugate net coincide in case $H=\mathfrak{S}, K=\Omega$. In this case the points (87) are the ray-points (82), and the point (88) is the point $y$ on the axis. Then the expansions (84), (29) automatically agree to terms of the fourth order.

* Green, Projective differential geometry of one-parameter families of space curves, and conjugate nets on a curved surface (Second Memoir), American Journal of Mathematics, vol. 38 (1916), p. 287.

† Lane, ibid., p. 147. 
The inverse of the transformation (30) of unit point, with $l, m, n$ given by (32), can be used to change the equations in the notation of $\S 3$, based on equation (45), to the notation based on equation (29) with arbitrary unit point. Thus, for example, equation (74) becomes

$$
a_{2} b_{0} x+a_{0} b_{3} y=0 \text {. }
$$

In the notation of the theory of conjugate nets this equation becomes

$$
\mathfrak{S}^{\prime} x+\mathfrak{B}^{\prime} y=0 \text {. }
$$

This is the line joining the point $x$ to the point of intersection of the ray and the associate ray, which has been called* by Davis the second canonical tangent of the conjugate net and its associate conjugate net. Thus the following theorem is proved.

The cone of Kubota and $S u$ associated with a tangent of a conjugate net at a point of a surface intersects the tangent plane, besides in the tangent itself, also in Davis's second canonical tangent at the point. p. 19.

* W. M. Davis, Contributions to the Theory of Conjugate Nets, Chicago Doctor's Thesis (1932),

University of Chicago,

Chicago, Ill. 